\title{
Using cluster analysis to identify patterns in students' responses to contextually different conceptual problems
}

\author{
John Stewart, ${ }^{*}$ Mayo Miller, Christine Audo, and Gay Stewart \\ Physics Department, University of Arkansas, Fayetteville, Arkansas 72701, USA
}

(Received 23 February 2012; published 4 October 2012)

\begin{abstract}
This study examined the evolution of student responses to seven contextually different versions of two Force Concept Inventory questions in an introductory physics course at the University of Arkansas. The consistency in answering the closely related questions evolved little over the seven-question exam. A model for the state of student knowledge involving the probability of selecting one of the multiple-choice answers was developed. Criteria for using clustering algorithms to extract model parameters were explored and it was found that the overlap between the probability distributions of the model vectors was an important parameter in characterizing the cluster models. The course data were then clustered and the extracted model showed that students largely fit into two groups both pre- and postinstruction: one that answered all questions correctly with high probability and one that selected the distracter representing the same misconception with high probability. For the course studied, $14 \%$ of the students were left with persistent misconceptions post instruction on a static force problem and $30 \%$ on a dynamic Newton's third law problem. These students selected the answer representing the predominant misconception slightly more consistently postinstruction, indicating that the course studied had been ineffective at moving this subgroup of students nearer a Newtonian force concept and had instead moved them slightly farther away from a correct conceptual understanding of these two problems. The consistency in answering pairs of problems with varied physical contexts is shown to be an important supplementary statistic to the score on the problems and suggests that the inclusion of such problem pairs in future conceptual inventories would be efficacious. Multiple, contextually varied questions further probe the structure of students' knowledge. To allow working instructors to make use of the additional insight gained from cluster analysis, it is our hope that the physics education research community will make these methods available though their Web sites.
\end{abstract}

DOI: 10.1103/PhysRevSTPER.8.020112

PACS numbers: 01.40.Di, 01.40.Fk

\section{INTRODUCTION}

Pre- and posttesting using research-validated instruments has become standard practice for evaluating the conceptual learning in introductory physics courses [1]. This practice gives valuable but incomplete information about the state of students' knowledge entering and leaving a physics course. For example, at the University of Arkansas the Spring 2006 Force Concept Inventory (FCI) [2] pretest yielded the following averages for the selection of the five responses to FCI problem 29: (a) 8\%, (b) $45 \%$, (c) $3 \%$, (d) $41 \%$, and (e) $3 \%$. The correct answer is (b). While informative, these average values lack important information which could be used to shape instructional decisions. Consider an experiment that asks a number of questions similar to FCI problem 29 (a problem involving the forces on a chair sitting on the floor, reproduced in the Appendix), where each question uses a different physical

\footnotetext{
*johns@uark.edu
}

Published by the American Physical Society under the terms of the Creative Commons Attribution 3.0 License. Further distribution of this work must maintain attribution to the author(s) and the published article's title, journal citation, and DOI. system. The averages above could be generated by a population of students where most students were wavering between the correct answer and the misconception represented by response (d). This population is applying both a Newtonian model and a novice model that produces the misconception represented by answer (d). The average could also be produced by a course where about half the students always select the correct answer (b) and where about half the students always select answer (d); the second set of students presumably is consistently applying the misconception represented by answer (d). These two populations of students, the always consistent population and the inconsistent population, have very different incoming knowledge states.

The consistency of student answers to problems where the underlying physical system is similar has been extensively investigated. There is substantial evidence that students do not leave most introductory physics courses with an integrated, expertlike understanding of the material [1-3]. This lack of expertlike understanding can take many forms, from persistent, consistently applied misconceptions to a sensitivity to the surface features of a problem such as the physical system or the presentation of the problem. In this work, the physical system used in a 
problem will be called its context. Students who answer differently to problems with different contexts have a sensitivity to the context. Related to the context, the form in which the problem is presented, whether the statement of the problem is mathematical, textual, diagrammatic, or pictorial, will be called the problem's representation.

This paper will investigate the evolution of consistency of students' answers over seven contextually related questions embedded in the FCI. Using multiple, contextually different questions with the same underlying physical interpretation is an important experimental technique used to investigate expert-novice differences, the structure of novice models, contextual and representational sensitivity, and the consistency of application of novice models. The survey of research that follows is a small sample of the applications of the technique.

The role of context has been important in the understanding of the structure of novice physics knowledge and the application of that knowledge to physical situations [4,5]. The difference in the methods of solution of problems by experts and novices has been investigated using contextually related questions. Students group mathematics and physics problems differently than experts, with expert problem solvers grouping problems based on their underlying physical or mathematical structure and novice problem solvers often grouping problems based on their surface features such as context or question format [6-8]. The representation-use pattern of novice and expert problem solvers also shows identifiable differences in the patterns of use of multiple representations [9].

Multiple, physically similar problems have been used to investigate how consistently alternate models are applied. Substantial variation in the consistency of the application of alternate frameworks and scientifically correct frameworks was found with students often using both the correct model and an alternate model in different situations [10-12]. The consistency of student reasoning (either correct or incorrect) was found to increase with additional instruction [12-14] and to depend on the familiarity of the context of the problems [15].

Since context sensitivity probes the degree to which a student population has developed an integrated understanding of a topic, it has been studied in a number of environments. Testing student understanding using multiple, contextually related questions has been used with physics problem pairs involving an inanimate and a living context [16], an abstract and a concrete context [17], and an abstract and a familiar context [18]. Students performed slightly better on problems where the context was familiar [18]. Multiple-question instruments are also used; Palmer investigated Newton's third law using a nine-question test involving a range of contexts from the "hard" contexts commonly found in physics problems such as tables or the ground to "soft" contexts less commonly found in physics problems such as water or a mattress [19].
Differences in performance based on problem representation have also been investigated. Novices react to surface features of problems involving their representation that would be unimportant to experts just as they respond to contextual surface features. McDermott et al. found students had difficulty in interpreting many features of kinematics graphs and designed interventions to address these problems and to foster the use of multiple representations [20]. These interventions use multiple contexts to augment graphical skill. Differences in the representation of physics problems were found to produce strong differences in performance [21-23]. Differences in performance based on representation were also influenced by the context of the problems [22,23]. Savinainen and co-workers constructed a conceptual instrument derived from the Force Concept Inventory to investigate representational coherence and found a substantial improvement in coherence between the pretest and posttest in high school students $[24,25]$. In mathematics education, work comparing performance on story, word, and symbolic problems seems to show superior performance on contextually richer story problems [26] (this work contains an extensive discussion of the issue of story problems).

The use of multiple representations $[27,28]$ and enriched contexts $[29,30]$ has also been used as part of instructional programs designed to produce more expertlike thinking or to improve the attitudes of students toward science. Application of contextually paired problems has also been investigated as an instructional method. The efficacy of this technique was found to depend strongly on how the paired problems were presented [31-33].

All of these studies use multiple, similar problems to investigate or improve student understanding, usually using pairs of problems but sometimes using many similar problems. This opens the possibility that the state of student knowledge evolves over the course of the experiment. The work of Rennie and Parker shows some slight improvement in results in the second of a pair of problems [18], but their sample size is so small $(N=8)$ that it is unlikely the observed effect is statistically significant.

The works described above use a variety of methodologies. Many use paired problems, but some use up to nine similar problems. The degree of the similarity of the problems also varies widely with some problems differing only by the textual description of the physical system, some problems differing by a rewriting of the problem using a graphical or mathematical representation to replace the problem text, and some problems differing widely related only by the underlying physical principles needed to solve the problem.

Overall, a few themes emerge. The state of student knowledge after a science course is complex, with students often holding multiple, conflicting models after instruction. The consistency of application of these models increases with additional education. The consistency is also 
influenced by the familiarity of the students with the context of the problem.

The studies above examined the consistency of students' answers in a variety of situations, but do little to develop more detailed models of the consistency of the student populations tested. Bao and Redish [34] introduced a framework, model analysis, for further exploring the structure of the consistency of the application of student knowledge by separating a group of students into intellectually similar subgroups. Approximate methods for extracting the size and structure of the subgroups was also developed. Bao and Redish [34] make a compelling argument for the need for further investigation into the structure of the knowledge state of the student population: "Analyses using scores alone often fail to provide useful details on the students' real understanding of the physics concept (except in the case when most students consistently give correct answers). For example, a low score can be caused by a consistent incorrect model, calculation errors generated while using a correct model, random guessing, or a persistently triggered incorrect model for a student in a mixed model state. These different situations reflect important information on student understanding of physics, but they cannot be distinguished using an analysis based solely on scores."

Model analysis has been used to investigate the structure of the consistency of student answers to conceptual problems in mechanics [34]. It was found that 74\%-82\% of the students were associated with a single subgroup for both the pretest and the posttest in both a course using traditional instruction and a course using modified instruction; however, the students in the nontraditional course much more consistently applied the correct model than the students in the traditional course. The method was also used to explore the structure of students' understanding of Newton's third law, and it was found that detailed features of Newton's third law problems involving whether the objects were stationary, moving, or accelerating generated student subgroups with very different properties [35].

The problem of taking a set of data and separating it into subgroups where the members of each subgroup are more similar to each other than they are to members not in the subgroup is a problem that has been extensively studied. One well-understood statistical method for performing such a separation is cluster analysis. Cluster analysis will converge to a set of subgroups that are within a preset tolerance of the mathematically best set of subgroups.

This study seeks to investigate the following questions:

(1) Does the consistency of students' answers evolve over the course of an exam?

(2) How can a data set containing the responses to multiple, contextually related questions be used to subdivide a class into intellectually similar subgroups?
(3) Once a model subdividing a class into groups is constructed, how does one evaluate the quality of the model?

(4) What are the subgroup models of the Spring 2006 and Fall 2006 University Physics courses at the University of Arkansas?.

\section{MEASUREMENT}

In 2006-2007, an experiment was performed at the University of Arkansas that investigated how context influenced the consistency of students' responses to conceptual physics questions. This study was conducted with students enrolled in the introductory calculus-based mechanics course during the Spring 2006 (147 students) and Fall 2006 (200 students) semesters. During the first day of physics laboratory, students were given a 36 question pretest using 30 Force Concept Inventory (FCI) questions mixed with six variations of one FCI question. The six variations modified the physical context of the FCI question.

Students who took the pretest received extra credit for participation, but additional extra credit was also possible based on superior performance. Later in the semester, the same exam was administered as a posttest; this posttest was part of the third exam (four exams total). Data from the pretests and posttests were excluded for students that did not complete the course.

The course used for the measurement, University Physics I, is the first-semester, calculus-based mechanics course in the introductory physics sequence for scientists and engineers. The course is presented with two 50-minute traditional lectures and two 110-minute laboratories per week. The laboratories are a mix of teaching assistant led problem solving, small group work, hands-on interactive laboratories, and traditional experiments. The course was developed as part of the University of Arkansas' Physics Teacher Education Coalition site and produced an average normalized gain on the FCI of 0.51 for the semesters studied.

Two versions of the modified FCI test were used, one for the Spring 2006 semester and one for the Fall 2006 semester. The Spring semester test contained the 30 FCI questions and six additional questions regarding the forces acting on a stationary object that were produced by modifying the context of FCI problem 29 . The original FCI problem and an example of a transformed problem are included in the Appendix. A different physical context was used for each problem. The new contexts varied from traditional physics "hard" contexts (marble resting on a table) to less traditional "soft" contexts (balloon floating in the air). Table I shows the contexts used. As much as possible, the context-modified problems were created by word substitution, replacing the original context with the modified context. The order of the multiplechoice responses was unchanged. This work uses the 
TABLE I. Problems' averages and consistency. $S$ is the average score on the problem and $C_{p}$ the average consistency.

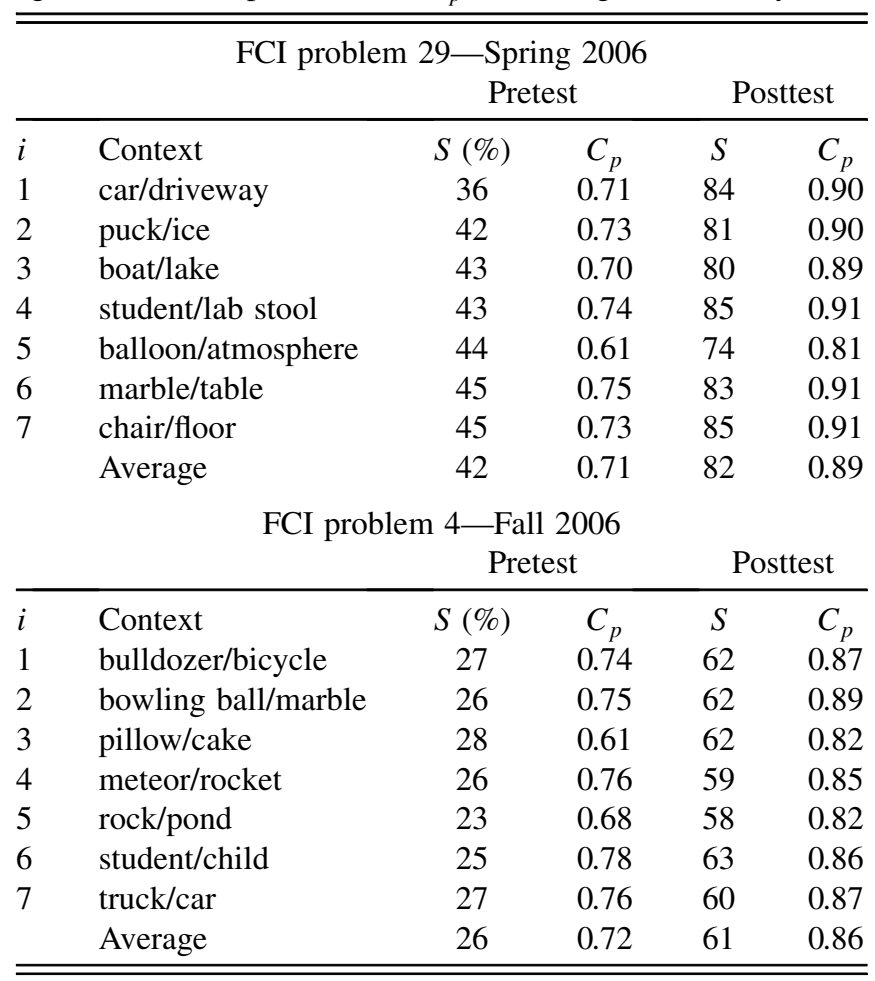

revised version of the FCI included with Mazur's Peer Instruction [36].

To reduce the risk of students recognizing similarity in the seven questions, they were spread throughout the test. For the Spring semester, the context-related questions were questions $3,9,15,20,24,30$, and 35 in the 36-question exam. Three versions of the test were created; the contextrelated questions were asked in the same locations, but the seven questions were ordered differently. The order of the problems in the three tests using the index, $i$, in Table I was test $1: 1,2,3,4,5,6,7$; test $2: 7,6,3,4,5,2,1$; test $3: 2,7$, $3,6,5,1,4$. Note that problems 3 and 5 were in the same location in all three tests. This was done because previous research suggested that the students' response to the unusual (for a physics course) contexts in the problems would generate a different response pattern [19]. By placing the problems in the same location, these effects could be eliminated if necessary. Each of the three versions of each test were given to approximately one-third of the students in each course.

The same procedure was followed with a different FCI question in Fall 2006. Six context-modified questions were written for FCI question 4, a question that involves the application of Newton's third law in a situation where at least one object is moving. The questions were placed in the same locations as in Spring 2006. Again, three versions of the test were formed using the same sequences as in Spring 2006.
FCI problems 4 and 29 were chosen for the experiment because each could be reliably transformed by simple word substitution since neither had an associated figure and neither was a member of a multiproblem group. Past experience with the FCI indicated that the pretest scores on problem 29 would be near $45 \%$ with posttest scores near $85 \%$ while the pretest and posttest scores on problem 4 would be substantially lower. This provided a set of results where the average on the set of problems had substantial variation.

\section{CONSISTENCY}

Consistency in answering represents a complementary dimension to the score as a characterization of the state of a student's understanding. For the $k$ th student, the consistency between the answers of problem $i$ and problem $j$ can be captured with the statistic $C_{i j}^{k}$, where $C_{i j}^{k}=1$ if the same response is selected on both problems and $C_{i j}^{k}=0$ otherwise. $C_{i j}^{k}$ is 1 if the student selected the correct answer for both problems or if the student selected the same incorrect answer. Figure 1 plots the average pairwise consistency $\left\langle C_{i, i+1}\right\rangle$ for each of the three test orders where the average is taken over all students taking one of the three differently ordered tests. We focus on the pretest consistency because the pretest scores show much more variability than the posttest scores.

The consistency $C_{p}$ of one of the problems $p$ in a multiproblem set can be defined as the average of $C_{i j}^{k}$ over the other problems and the set of students,

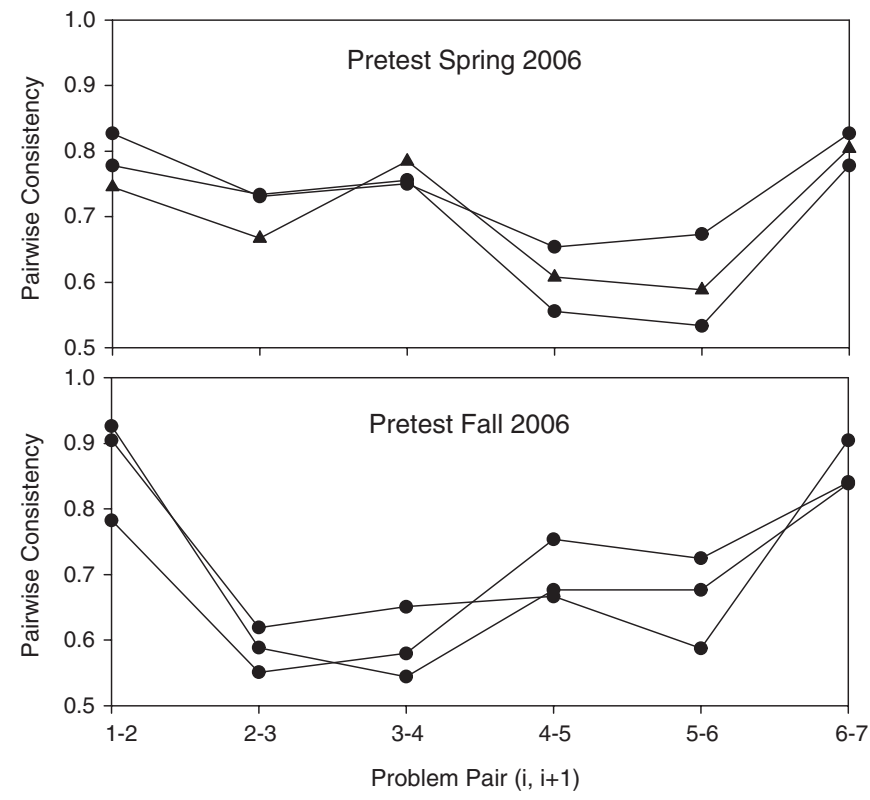

FIG. 1. Consistency of student response to pretest questions. The average pairwise consistency $\left\langle C_{i, i+1}\right\rangle$ is plotted against the location of the pair of problems in the test. The three lines represent the three orders of the problems. 


$$
C_{p}=\frac{1}{N} \frac{1}{N_{q}-1} \sum_{k=1}^{N} \sum_{i \neq p}^{N_{q}} C_{i p}^{k}
$$

where $N$ is the number of students and $N_{q}=7$ the number of contextually related questions. The average consistency $C$ over a set of problems can then be defined as the average of $C_{p}$. Table I shows the score $S$ and the consistency $C_{p}$ of each problem and the average score and consistency of all problems.

The consistency is not free to independently vary from 0 to 1 . As the score $S$ on a question increases, the consistency should also tend to increase. If $S=100 \%$, then $C=1$.

Figure 1 plots the average consistency per problem pair $\left\langle C_{i, i+1}\right\rangle$ versus the relative location of the pair $i$ within the test. These figures show little evidence of increasing consistency as students answer more, related questions.

There is little variation in pretest averages $S$, with only question 1 in Spring 2006 scoring substantially lower than the other questions. There is some evidence in the pretest data that the students answered less consistently for contexts that were "soft," question 5 in Spring 2006 and questions 3 and 5 in Fall 2006. This difference in consistency largely vanishes postinstruction with only question 5 in Spring 2006 answered substantially less consistently than the other questions. The varying response of students to the soft contexts lends support to Palmer's observation of substantial variation on an instrument using multiple, soft contexts [19].

The shape of the curves in Fig. 1 largely results from the variation in consistency of the problems discussed above and not from the location of the problem in the test, as problems 3 and 5 remained in the same location.
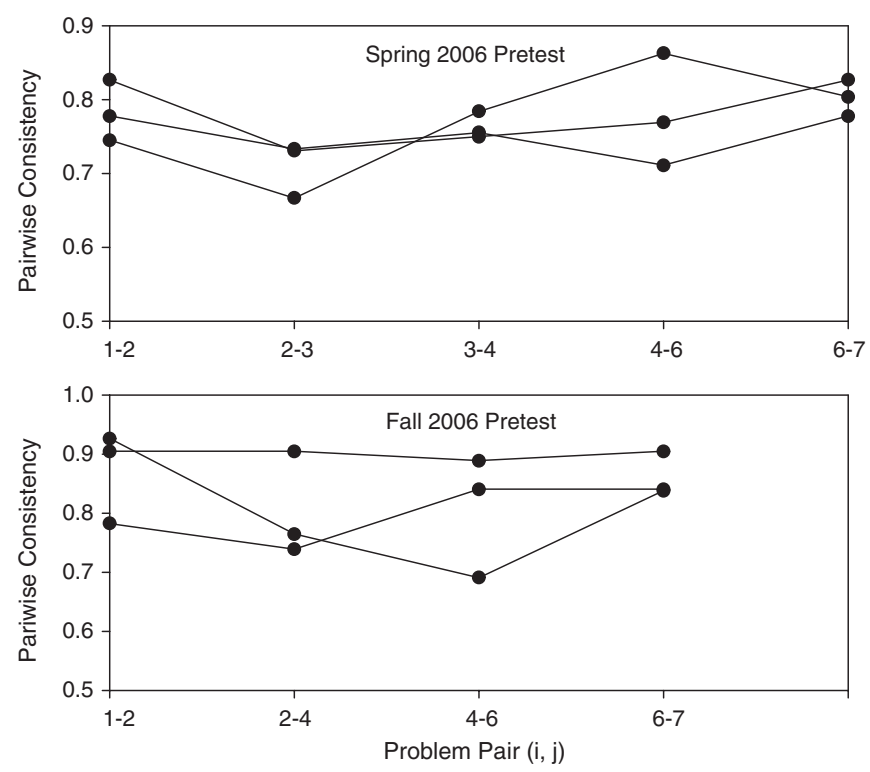

FIG. 2. Consistency of student response to pretest questions with question 5 from Spring 2006 and questions 3 and 5 from Fall 2006 removed.
A linear fit to the Spring 2006 pretest data yielded $\left\langle C_{i, i+1}\right\rangle=-0.011 i+0.75$. A similar fit to the Fall 2006 pretest data gives $\left\langle C_{i, i+1}\right\rangle=-0.001 i+0.68$. The consistency of student answers decreased slightly as the students answered more contextually related questions.

Figure 2 plots the consistency with problem 5 of Spring 2006 and problems 3 and 5 of Fall 2006 removed. Linear fits to the average consistency with these problems removed yielded $\left\langle C_{i, i+1}\right\rangle=0.011 i+0.73$ for Spring 2006 pretest and $\left\langle C_{i, i+1}\right\rangle=-0.013 i+0.84$ for Fall 2006. Using either the complete set of problems or the reduced set, the change in consistency over the seven problems is very small, around $1 \%$ per problem. This slight improvement as additional problems are worked is consistent with the observations of Rennie and Parker [18].

\section{PROBABILISTIC MODEL}

The set of seven contextually related questions generates a sequence of seven responses for each student, for example, $a, a, a, c, a, b, a$, where each letter represents the answer choice selected. The pairwise consistency $C_{i, i+1}$ changed little over the course of the test. This suggests that even though the contextually related questions are quite similar, illustrating the same physical principle and having a response set that is the same up to the change in context, the students' pattern of answering the first question is approximately the same as for the last question. As such, the order of the questions is not an important factor in how a student answers, so the response of a student can be modeled as a response vector $\vec{R}$, where each component counts the number of times a particular answer choice was made. For the example given above, $\vec{R}=(5,1,1,0,0)$, indicating that response $a$ was selected five times, responses $b$ and $c$ once, and responses $d$ and $e$ were not selected. The response vectors that resulted from the Spring 2006 pretest data are shown in Fig. 3, the vectors from the Fall 2006 pretest data in Fig. 4, the vectors from the Spring 2006 posttest data in Fig. 5, and the vectors from the Fall 2006 posttest data in Fig. 6. In all figures, the response vector $(5,1,1,0,0)$ is abbreviated as 51100 .

The vectors shown in Figs. 3 and 4 are quite different than would appear by random chance. There are

$$
N_{v}=\frac{\left(N_{r}+N_{q}\right) !}{\left(N_{r}-1\right) ! N_{q} !}=330
$$

possible vectors for an experiment with $N_{q}=7$ questions each with $N_{r}=5$ possible responses. Only 44 of the possible vectors are present in the Spring 2006 pretest and 41 in the Fall 2006 pretest. Many vectors appear only one or two times, but there are also strong peaks in the data, particularly for students who answer completely consistently either always correct, 07000 (Spring 2006) and 00007 (Fall 2006), or always select the same incorrect response, 00070 (Spring 2006) and 70000 (Fall 2006). In 


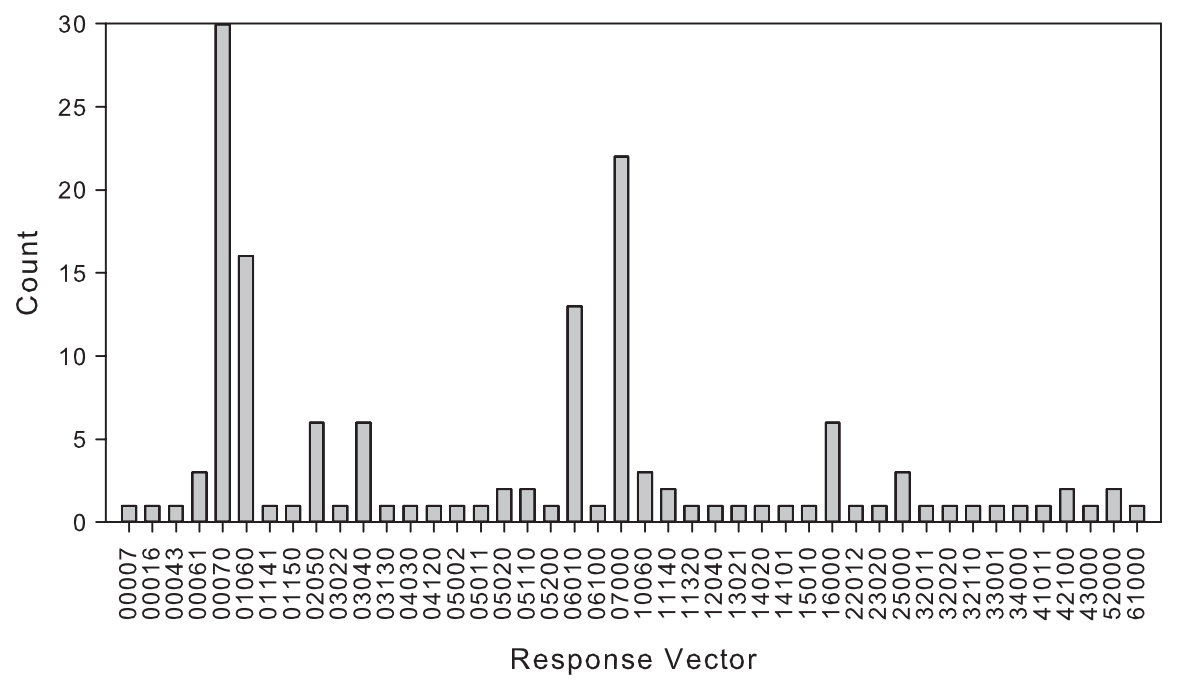

FIG. 3. Distribution of response vectors for Spring 2006 pretest data.

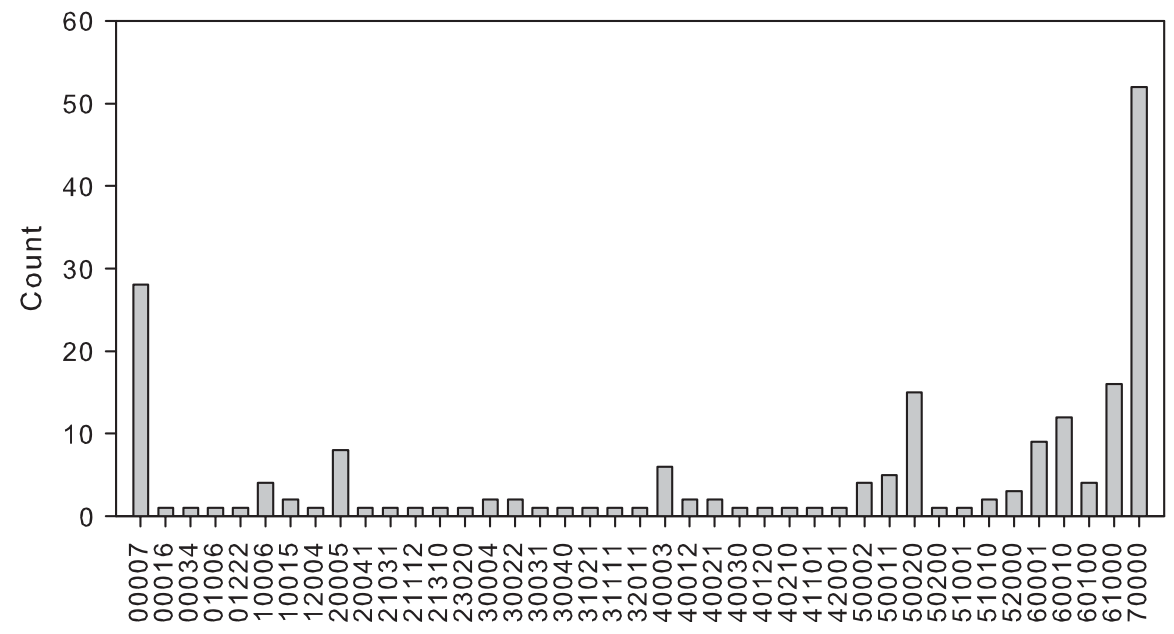

Response Vector

FIG. 4. Distribution of response vectors for Fall 2006 pretest data.

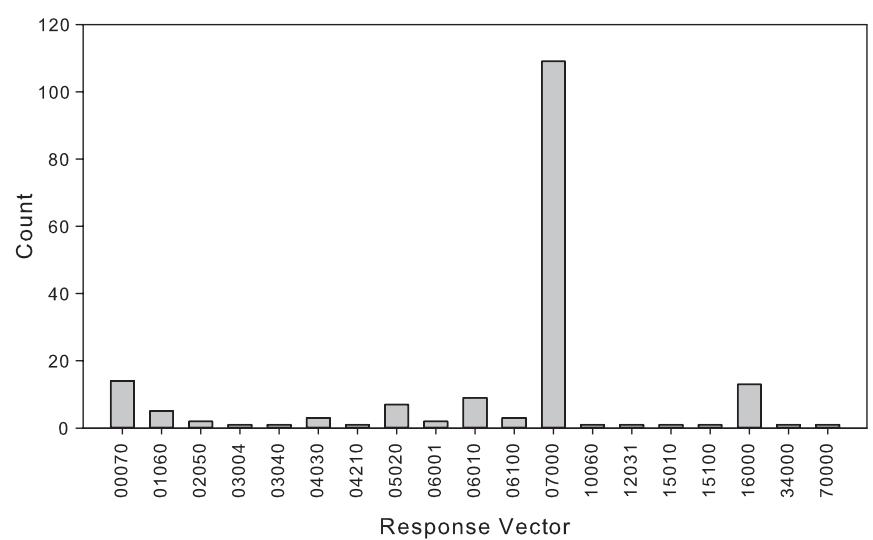

FIG. 5. Distribution of response vectors for Spring 2006 posttest data.

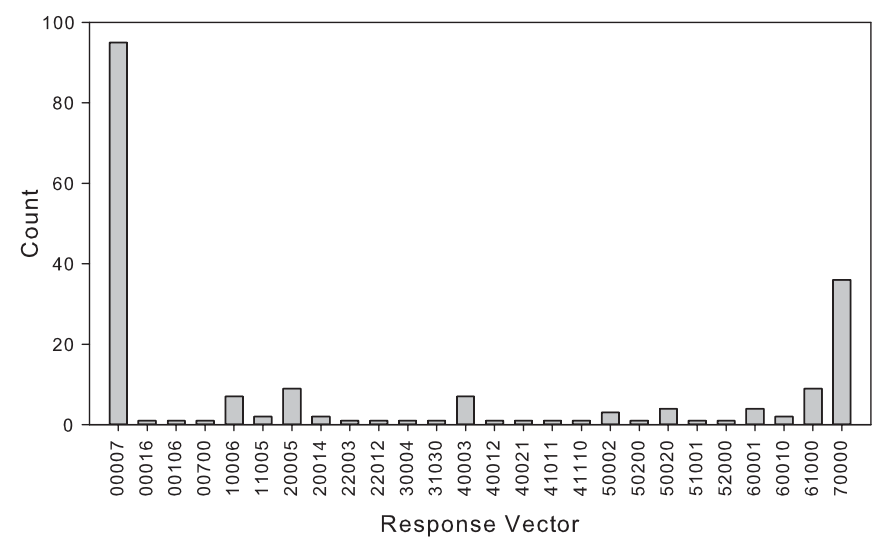

FIG. 6. Distribution of response vectors for Fall 2006 posttest data. 
the Spring 2006 vectors, there is some evidence of a small group who are uncertain about which answer to select between answer (b) and answer (d) as shown by the peaks at 02050 and 03040.

Since the students' response pattern does not vary significantly as additional contextually related problems are asked, one potential model of the students' knowledge measured by the question set would be a model giving the students' probability of selecting each answer. This probability can be represented as a vector $\vec{p}=$ $\left(p_{1}, p_{2}, p_{3}, p_{4}, p_{5}\right)$, where $p_{1}$ is the probability the student selects the first response, response $a$. The probability vector $\vec{p}$ for a student can be estimated from the response vector for that student as $\vec{p}=\vec{R} / N_{q}=\left(R_{1} / N_{q}\right.$, $\left.R_{2} / N_{q}, R_{3} / N_{q}, R_{4} / N_{q}, R_{5} / N_{q}\right)$.

The distribution of response vectors shown in Figs. 3 and 4 seems to indicate that there are subgroups of students with similar answering patterns. Focusing on the Spring 2006 data set, the peaks near the correct answer 07000 including vectors 07000,06010 , and 16000 give evidence for a subgroup of students with probability vector $\vec{p}=$ $(\gamma, \alpha, 0, \beta, 0)$, where $\alpha \gg \beta>\gamma$. Note that this example illustrates a danger in the response vector plots. The response vectors are five dimensional; a plot like Fig. 3, which presents the five-dimensional object on a onedimensional graph, often does not represent the distance between two vectors correctly. The peaks at 00070, 01060, and 00061 suggest a second group with $\vec{p}$ near $(0, \beta, 0, \alpha, \gamma)$, where $\alpha \gg \beta>\gamma$. The vectors 02050 and 03040 give some evidence for smaller third group with $\vec{p}$ near $(0, \beta, 0, \alpha, 0)$ with $\alpha>\beta$. The cluster analysis performed in Sec. VII will further support the existence of this subgroup.

As a result of the above discussion, it seems reasonable to try to extract a model of the state of student knowledge for the pretest and posttest data in terms of a set of subgroups of students where each student in a subgroup has the same probability vector $\vec{p}_{i}$. The size of the subgroups will be characterized by $\gamma_{i}=N_{i} / N$, which will be called the group population fraction, where $N_{i}$ is the number of students in the group and $N$ is the number of students in the course, $N=\sum_{i} N_{i}$.

\section{A. Overlap}

Later in the paper we will examine the problem of extracting $\gamma_{i}$ and $\vec{p}_{i}$ from a data set and characterizing the reliability of the extraction. A key property that determines whether two subgroups can be resolved is the geometric overlap of the average radius of the sphere that contains the subgroups. The subgroups in this paper are modeled with vectors representing the probability of choosing a certain response to a physics question. The overlap of two subgroups is then related to the probability that two students from different subgroups generate the same response vector. This probability will be called the overlap, $V_{i j}$, between the two subgroups and can be calculated directly from $\vec{p}_{i}$.

The probability that a student with probability vector $\vec{p}=\left(p_{1}, p_{2}, p_{3}, p_{4}, p_{5}\right)$ will generate the response vector $\vec{R}=\left(R_{1}, R_{2}, R_{3}, R_{4}, R_{5}\right)$ is defined as $\mathcal{P}(\vec{R} ; \vec{p})$. For an $N_{q}$ question experiment where each question has $N_{r}$ responses, $\mathcal{P}(\vec{R} ; \vec{p})$ is given by

$$
\mathcal{P}(\vec{R} ; \vec{p})=\left(\frac{N_{q} !}{R_{1} ! R_{2} ! R_{3} ! R_{4} ! R_{5} !}\right) p_{1}^{R_{1}} p_{2}^{R_{2}} p_{3}^{R_{3}} p_{4}^{R_{4}} p_{5}^{R_{5}} .
$$

The overlap between the probability distributions of vector $\vec{p}_{i}$ and $\vec{p}_{j}$ can then be defined as

$$
V_{i j}=\sum_{\vec{R}} \min \left(\mathcal{P}\left(\vec{R} ; \vec{p}_{i}\right), \mathcal{P}\left(\vec{R} ; \vec{p}_{j}\right)\right),
$$

where the sum is taken over all response vectors. If the probability distributions were continuous, the overlap would be the area under both probability curves. The overlap $V$ of a model will be defined as the maximum pairwise overlap of the vectors in the model.

\section{B. Consistency}

With the probability of each vector given in Eq. (3) one can calculate the consistency $C^{k}$ of a student with probability vector $\vec{p}^{k}$. As before, define the consistency of student $k$ on two related problems, $i$ and $j$, as $C_{i j}^{k}$ where $C_{i j}^{k}$ is 1 if the problems are answered consistently and 0 otherwise. The consistency of a student is then the average of $C_{i j}^{k}, C^{k}=\left\langle C_{i j}^{k}\right\rangle$, over the set of problem pairs. Likewise, the consistency of a course can be defined as the average of the consistency of its students. The consistency $C^{k}$ is the probability of student $k$ selecting the same answer in a twoquestion experiment. Using $N_{q}=2$ and $\vec{R}^{k}=(2,0,0,0,0)$ in Eq. (3) yields the probability that the first multiplechoice answer is selected for both questions in a two-question experiment as $\left(p_{1}^{k}\right)^{2}$. The consistency of a student with probability vector $\vec{p}^{k}, C^{k}$, is then

$$
C^{k}=\vec{p}^{k} \cdot \vec{p}^{k}
$$

The consistency of a course $C$ with group population fractions $\gamma_{i}$ is

$$
C=\sum_{i} \gamma_{i} \vec{p}_{i} \cdot \vec{p}_{i}
$$

\section{Symmetric two-component vectors}

To investigate the properties of a set of probability vectors that would generate a data set that can be reliably divided into subgroups, consider the probability vectors and group population fractions for the two-group model:

$$
\begin{array}{ll}
\gamma_{1}=0.5, & \vec{p}_{1}=(a, 1-a, 0,0,0), \\
\gamma_{2}=0.5, & \vec{p}_{2}=(1-a, a, 0,0,0) .
\end{array}
$$


This model depends on one parameter, $a$, and produces a symmetric response vector probability distribution. For $N_{q}=7$, there are only eight response vectors produced by this model. The probability distributions for $a=0.95$, $a=0.8, a=0.75$, and $a=0.7$ are shown in Fig. 7, where the probability is plotted in terms of $N_{1}$, the number of times a student selects the first response choice. The overlap $V$ is calculated for each figure.

Figure 7 shows that for models with small overlap $(V \approx 0)$ two easily identifiable subgroups exist. As the overlap increases, the peaks move together and eventually at $V=0.25$ it becomes difficult to visually resolve two distinct subgroups. At sufficiently high overlap, the division of the data into subgroups is not warranted.
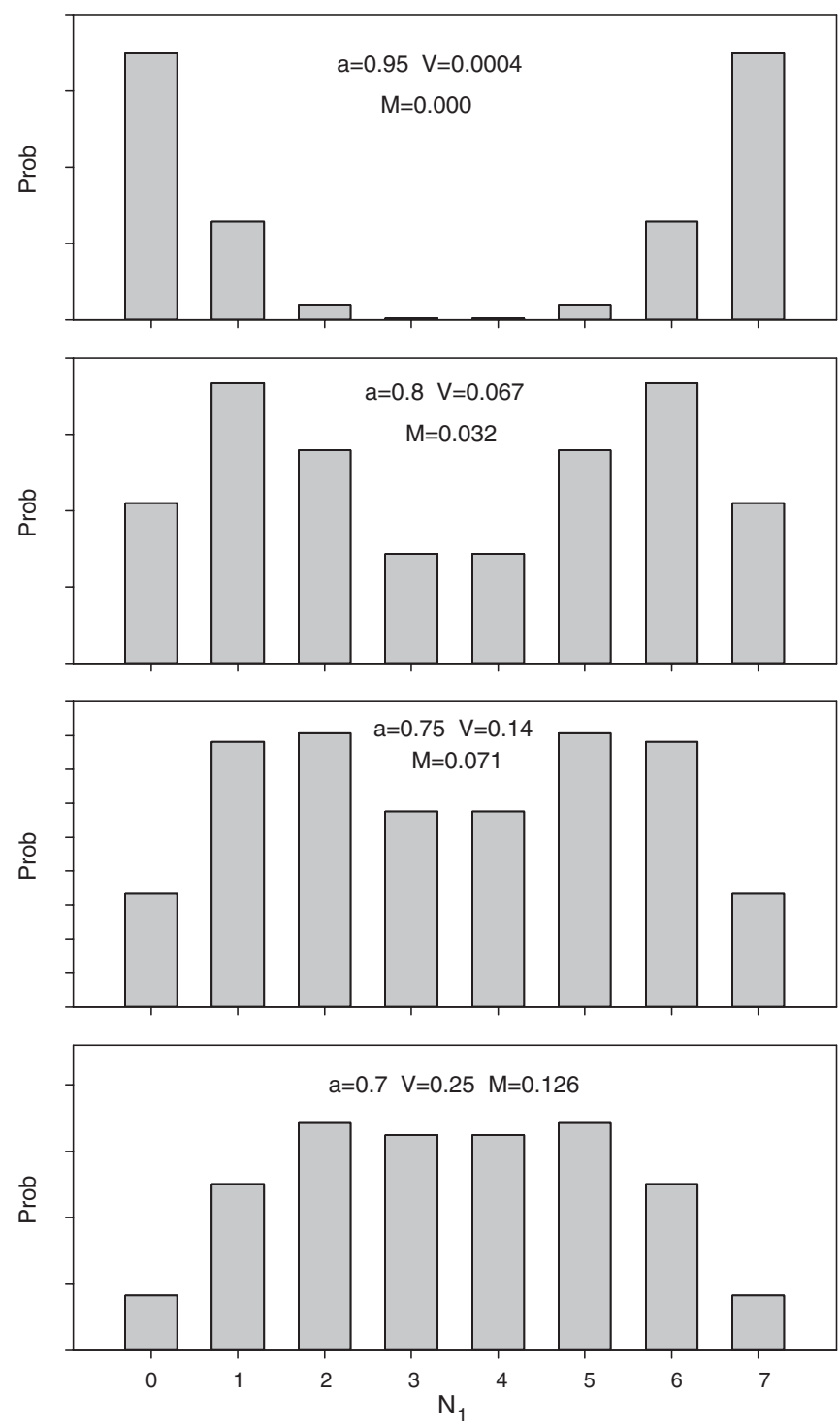

FIG. 7. Probability distribution of the response vectors of symmetric probability vectors. $V$ is the overlap between the two vectors in the model. $M$ is the misassignment rate in a simulated clustering experiment.

\section{COMPARISON OF SUBGROUP IDENTIFICATION METHODS}

Following the statistical analysis of Sec. IV, we would like to further our understanding of the 2006 course data by developing a model of the knowledge state of the students in the course in terms of a set of group population fractions $\gamma_{i}$ and probability vectors $\vec{p}_{i}$. The visual investigation of Figs. 3 and 4 provided a qualitative summary of the properties of both semesters' pretest data. This qualitative investigation does not, however, allow the estimation of the sizes of the subgroups of students or allow the exact characterization of the vectors of the subgroups. As with any visual investigation of a statistical data set, it is also possible that identifiable subgroups were missed or statistical fluctuations were spuriously identified as subgroups.

The additional detail offered by a model of the subgroup structure of a course will allow the investigation of important questions that cannot be answered by the average score or the average consistency. The course studied increased both the consistency and the score on the two problems considered, but what was the character of the groups of students that answered mostly correctly? Were they very sure of the correct answer, selecting correctly with high probability, or only mostly sure, periodically selecting an incorrect answer? How did the character of the mostly sure group (which was also present before instruction) change with instruction? Did the students who already had a Newtonian model apply it with higher probability after instruction? What was the character of the subgroup that still retained the alternate model? After instruction, did this group now apply the correct model and the alternate model with substantial probability? Did the noise seen in the response vector plots represent a group of students who were uncertain, oscillating between many answers? The subgroup models constructed below will answer all these questions and provide a remarkably detailed picture of the course studied.

Two very different methods will be considered for the extraction of an accurate set of group population fractions and probability vectors, model analysis [34] and cluster analysis. The purpose of this section is to compare the two methods and to select the best method to use to model the 2006 data.

Model analysis provides a computationally efficient method for extracting $\gamma_{i}$ and $\vec{p}_{i}$ based on an analogy with the quantum mechanical density matrix. Unfortunately, it is only exact in certain limits and does not allow the control of the number of subgroups extracted. Since model analysis is only used within the physics education research community, it is not as extensively studied as clustering algorithms and as such is not supported in statistical software packages.

Cluster analysis is broadly studied and is a common technique in many disciplines. Some clustering algorithms allow for the control of the number of clusters, subgroups, 
in the model and provide models that can be made arbitrarily close to the maximal $R^{2}$ model (defined below). Since clustering algorithms are common, most statistical software packages contain a variety of algorithms. The algorithm investigated in Sec. VB, $k$-means, was chosen for its simplicity, its long history, its control of the number of clusters, its broad acceptance, and its ease of implementation and modification. Many other algorithms are available and would yield similar results.

\section{A. Model analysis}

Model analysis was introduced by Bao in [37] and described by Bao and Redish [34] as a method for using multiple, matched problems measuring the same physical concept to develop a model of the state of student knowledge in terms of course model vectors, representing the knowledge state of subsets of the students in the course. The size of these subsets can be estimated using the eigenvalues of the course density matrix. This estimate is expected to be most accurate either when there is one dominant subgroup, one group with $\gamma_{i} \approx 1$, or when the probability vectors of the subgroups are orthogonal. Since the $\gamma_{i}$ and $\vec{p}_{i}$ calculated using model analysis are approximate away from these two limiting cases, it is productive to investigate how rapidly the estimated values diverge from the exact values for classes not meeting either of these conditions. The formalism introduced in Sec. IV offers a rich platform for investigating model analysis. The following uses the notation introduced by Bao in [37] and summarized in Bao et al. [35].

The student response vectors introduced in Sec. IV, $\vec{R}^{k}=\left(N_{1}^{k}, N_{2}^{k}, N_{3}^{k}, N_{4}^{k}, N_{5}^{k}\right)$, where $k$ indexes the student, can be converted into the single-student model state as

$$
\left|u_{k}\right\rangle=\frac{1}{\sqrt{N_{q}}}\left(\begin{array}{c}
\sqrt{N_{1}^{k}} \\
\sqrt{N_{2}^{k}} \\
\sqrt{N_{3}^{k}} \\
\sqrt{N_{4}^{k}} \\
\sqrt{N_{5}^{k}}
\end{array}\right),
$$

where $N_{q}=\sum N_{i}$ is the number of questions. The course density matrix is then formed as

$$
\mathcal{D}=\frac{1}{N} \sum_{k}\left|u_{k}\right\rangle\left\langle u_{k}\right|
$$

where $N$ is the number of students.

We will restrict our investigation to systems where each question has only two responses. With this simplification, the single-student model state vector becomes

$$
\left|u_{k}\right\rangle=\frac{1}{\sqrt{N_{q}}}\left(\begin{array}{c}
\sqrt{N_{1}^{k}} \\
\sqrt{N_{q}-N_{1}^{k}}
\end{array}\right) .
$$

Suppose the underlying state of knowledge of the students is represented by the probability vector $\vec{p}=(a, 1-a)$. In the large $N_{q}$ limit, where the students answer many similar questions, the single-student model state vector should converge to

$$
\left|u_{k}\right\rangle=\left(\begin{array}{c}
\sqrt{a} \\
\sqrt{1-a}
\end{array}\right)
$$

If all students have the same knowledge state, $\vec{p}=$ $(a, 1-a)$, the course density matrix becomes

$$
\mathcal{D}(a)=\left(\begin{array}{cc}
a & \sqrt{a-a^{2}} \\
\sqrt{a-a^{2}} & 1-a
\end{array}\right) .
$$

Model analysis predicts that the eigenvalues of the density matrix will be related to the group population fractions $\gamma_{i}$. If one computes the eigenvalues, $\gamma_{1}$ and $\gamma_{2}$, of $\mathcal{D}(a)$ in Eq. (12), one finds correctly that $\gamma_{1}=1$ and $\gamma_{2}=0$. So in the first case discussed above where one group dominates, model analysis recovers it [34].

Now consider a two-group model, Eq. (13), using symmetric probability vectors. This is a generalization of the system investigated in Sec. IV C:

$$
\begin{aligned}
& \gamma_{1}=\gamma, \quad \vec{p}_{1}=(a, 1-a), \\
& \gamma_{2}=1-\gamma, \quad \vec{p}_{2}=(1-a, a),
\end{aligned}
$$

This system produces the density matrix

$$
\begin{aligned}
\mathcal{D}(a, \gamma)= & \gamma\left(\begin{array}{cc}
a & \sqrt{a-a^{2}} \\
\sqrt{a-a^{2}} & 1-a
\end{array}\right) \\
& +(1-\gamma)\left(\begin{array}{cc}
1-a & \sqrt{a-a^{2}} \\
\sqrt{a-a^{2}} & a
\end{array}\right),
\end{aligned}
$$

which can be written as

$$
\mathcal{D}(a, \gamma)=\left(\begin{array}{cc}
1-b & \sqrt{a-a^{2}} \\
\sqrt{a-a^{2}} & b
\end{array}\right),
$$

where $b=a+\gamma-2 \gamma a$.

The general form of the eigenvalues of Eq. (14) is

$$
\gamma_{ \pm}=\frac{1}{2}\left(1 \pm \sqrt{4\left(a-a^{2}\right)-4\left(b-b^{2}\right)+1}\right) .
$$

To recover the model in Eq. (13), this expression should yield $\gamma_{ \pm}=\gamma$ and $1-\gamma$, which it does only when $a=1$ or $a=0$. If $a=0$ or $a=1$, the $\vec{p}_{i}$ vectors are orthogonal, so for the second limiting case presented above model, analysis correctly extracts $\gamma_{i}$ for this model.

Away from the two limiting cases, the $\gamma$ estimated using model analysis diverges from the $\gamma$ of the model, 


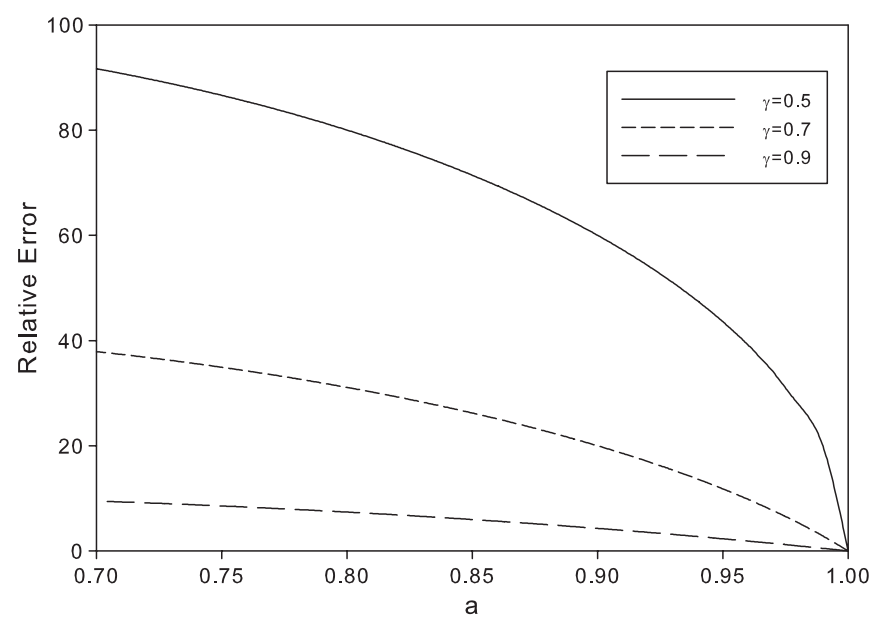

FIG. 8. Relative error vs $a$. The relative error $100 \% \times$ $\left(\gamma-\gamma_{+}\right) / \gamma$ vs $a$ where $\gamma_{+}$is the model analysis prediction and $\gamma$ is the exact value.

sometimes severely. Define the relative error in the model analysis estimate as

$$
\text { Relative error }=\left|\frac{\gamma_{+}-\gamma}{\gamma}\right| \times 100 \% \text {. }
$$

Figure 8 plots the relative error as a function of $a$ for $\gamma=0.5,0.7$, and 0.9. The figure shows that in the two limits $\gamma \rightarrow 1$ and $a \rightarrow 1$, the $\gamma_{+}$predicted by model analysis converges to the $\gamma$ used to build the model. Away from these limits $\gamma$ is consistently overestimated, particularly if the two groups have similar sizes.

Equation (16) can be used to estimate the relative error in the model analysis estimates of the group population fractions for the 2006 course data that are investigated using cluster analysis in Sec. VII. Using the largest group population fraction for $\gamma$ and the largest component of the group probability vector for $a$ extracted by cluster analysis, model analysis would produce a relative error of $85 \%$ for the Spring 2006 pretest, 4\% for the Spring 2006 posttest, 23\% for the Fall 2006 pretest, and 21\% for the Fall 2006 posttest. Bao and Redish suggest that for classes with maximum group population fractions below $\gamma=0.65$ additional methods beyond model analysis such as cluster analysis may be required [34].

\section{B. Cluster analysis}

Cluster analysis is a statistical method for constructing a subdivision of a data set that is broadly used over many disciplines. There are many algorithms for accomplishing the clustering. We will restrict our investigation to $k$-means clustering because of its conceptual simplicity, its availability in standard statistical software packages, and its ease of implementation as a computer program.

$k$-means is a widely used partitional clustering technique. The $k$-means method was first introduced in 1956, but was not given the name until 1967 [38]. The theory behind $k$-means clustering is very straightforward: given a data set $\vec{x}^{i}$ and a number of clusters $N_{c}$, partition the data set into $N_{c}$ subsets, $S_{j}$, such that the within cluster sum of squares (WCSS) is minimized:

$$
\mathrm{WCSS}=\sum_{j=1 . . N_{c}} \sum_{i \in S_{j}}\left(\vec{x}^{i}-\vec{c}^{j}\right) \cdot\left(\vec{x}^{i}-\vec{c}^{j}\right) .
$$

The vector $\vec{c}^{j}$ is the centroid of cluster $j$ defined as

$$
\vec{c}^{j}=\frac{1}{N_{c j}} \sum_{i \in S_{j}} \vec{x}^{i}
$$

where $N_{c j}$ is the number of members of cluster $j$. An effective $R^{2}$ for the cluster model can be calculated as

$$
R^{2}=1-\frac{\mathrm{WCSS}}{\mathrm{TSS}},
$$

where TSS is the total sum of squares calculated as the sum of the square distances from the overall average of the data.

The $k$-means algorithm begins with a random assignment of the members of the data set to the $N_{c}$ clusters. The centroid of each cluster is computed, then the Euclidean distance of each data point to each centroid is computed. Each data point is then moved to the cluster with the nearest centroid. The centroids are then recalculated and the process is iterated until the location of the centroids is constant within a predefined tolerance.

For the clustering experiments that follow, initial experimentation was carried out using MATLAB's implementation of the $k$-means algorithm. To improve performance, the $k$-means algorithm was then implemented in " $\mathrm{C}$ " and the new algorithm tested against MATLAB. The results of both algorithms were identical within tolerance.

The clusters selected by the $k$-means algorithm are dependent on the initial randomly selected centroids. To ensure that a true maximal $R^{2}$ model is found, the algorithm must be run multiple times and the best model selected from the multiple runs. $k$-means was used to cluster random data to determine the required number of iterations. The probability a single run would produce a model within some tolerance of the maximal $R^{2}$ model was weakly dependent on the number of points clustered, but more strongly dependent on the number of clusters selected. For a confidence of $95 \%$ that one of the iterations was within 0.005 of the maximum $R^{2}$ model, $k$-means was run 10 times for two clusters, 15 times for three clusters, 20 times for four clusters, and 25 times for five clusters.

To compare the use of model analysis and cluster analysis to calculate the relative sizes of student subgroups when $a \neq 1$, data sets were generated with $N=3000$ for $a=$ 0.7 to $a=0.95$ for the two-group model presented in Eq. (13). A large $N$ was used to allow comparison with the theoretical results of the previous section. Ten data sets were generated for each $a$ and results averaged. This small number of data sets was necessitated by the computational time involved in clustering the data sets. 


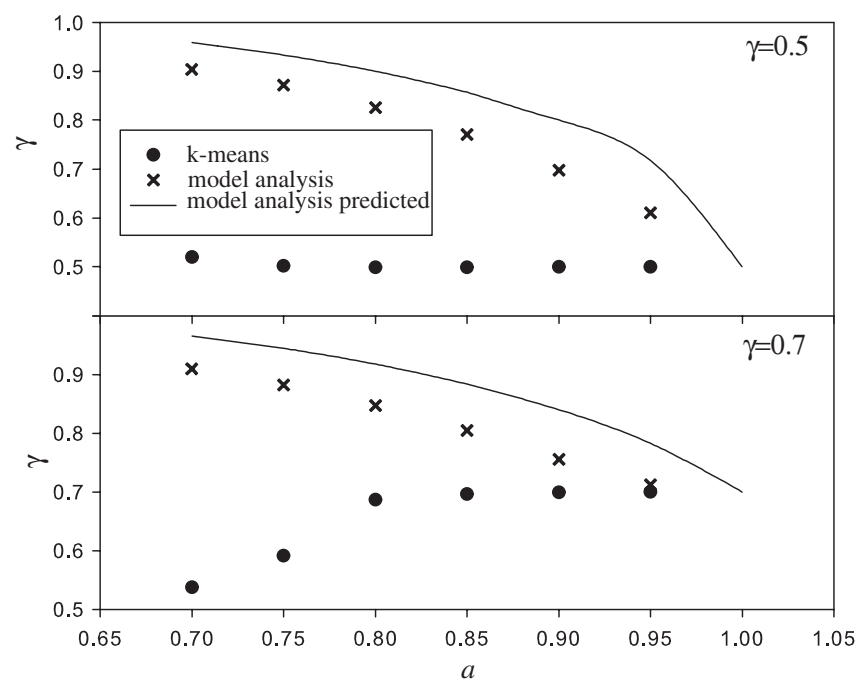

FIG. 9. Computed group population fraction $\gamma$ versus $a$. The data used in the top figure are generated with $\gamma=0.5$ and the bottom figure $\gamma=0.7$. The model analysis predicted line plots the theoretical prediction of the group population fraction calculated from Eq. (15).

Figure 9 plots the $\gamma$ computed by $k$-means and by model analysis against $a$ for $\gamma=0.5$ and $\gamma=0.7$. The theoretical $\gamma$ calculated from model analysis in the $N \rightarrow \infty$ limit given in Eq. (15) is also plotted. To perfectly recover the model used to generate the data, each curve should be a horizontal line passing through $\gamma$. The $k$-means algorithm does exceptionally well except for $\gamma=0.7$ and $a \leq 0.75$. The divergence from $\gamma=0.7$ for these cases may result from the small number of data sets averaged and the growth in error with increasing overlap. Model analysis correctly extracts $\gamma$ only for $a=1$ and then progressively diverges from the correct $\gamma$ as predicted in the previous section.

While model analysis is somewhat less computationally expensive than $k$-means or other traditional clustering algorithms, the group population fractions predicted by $k$-means more accurately reproduce the group population fractions of the underlying models except in the case where $a=1$. As such, to use model analysis one must be able to argue either that the data set is near the $\gamma=1$ limit or that the underlying model vectors are orthogonal. Since these conditions were not met for all course data under consideration, only cluster analysis will be used to analyze the 2006 course data.

\section{CHARACTERIZING COURSE MODELS}

$k$-means will generate a cluster model for any $N_{c}$ up to the number of data points. It is therefore important to be able to evaluate the fitness of a given model. The most important feature of a cluster model is that it explains a substantial amount of the variation in the data; good models have a large $R^{2} . R^{2}$ increases as the number of clusters increases and therefore a maximal $R^{2}$ value cannot be used to select an optimal model.
A second criterion is that the model correctly represents the underlying population. Error in representation occurs through a number of mechanisms; there can be error in the group population fraction $\gamma$ or in the probability vectors $\vec{p}$ extracted by the clustering algorithm. The clustering algorithm also partitions the data and may assign students to incorrect clusters.

The third criterion for a good cluster model is that the number of clusters is correct. Selecting the number of clusters in a set of data is an extremely active area of research. We experimented with a number of cluster selection criteria such as the "knee" method or the average silhouette. For the very discrete data produced by the seven-question experiment considered above, where there are only 330 possible data points, all these methods were extremely ambiguous.

Unlike in general cluster analysis, the centroids of the clusters that will be extracted below will be probability vectors. As was qualitatively explored in Sec. IV C, the overlap $V$ between two probability vectors will be a useful additional statistic for characterizing the degree to which a data set can be accurately separated into a set of clusters.

\section{A. Simulation experiments}

To understand the error in the clustering of student data, group population fractions $\gamma_{i}$ and probability vectors $\vec{p}_{i}$ were randomly generated for $N_{g}=2-5$, where $N_{g}$ is the number of groups. The generated $\gamma_{i}$ and $\vec{p}_{i}$ were then used to create a data set containing $N=300$ response vectors. This number of response vectors was selected as a reasonable size for an introductory science course at many universities. The effect of $N$ on the clustering error will be investigated in Sec. VIC.

Four strategies were used to generate the random models; for each strategy, 100000 data sets were created, clustered, and analyzed. Each strategy first randomly selected $N_{g}=2-5$ groups. With this selection, group population fractions $\gamma_{i}$ were randomly generated such that $\sum_{i} \gamma_{i}=1$. If the $\gamma_{i}$ selected would on average produce a group with less than 10 students, then $\gamma_{i}$ were regenerated. The first strategy then randomly generated the probability vectors $\vec{p}_{i}$. It was found that this generated models where the vectors were on average fairly close together. To produce models with vectors that were farther apart, three additional strategies were used. The second strategy calculated the total probability already allocated to previously generated probability vectors $p_{t}$ and weighted the probability of that component of the next vector by $1-p_{t}$. The third strategy randomly generated vectors as before, but required that $\vec{p}$ have only two nonzero components. The last strategy modified strategy three and produced only vectors with three nonzero components. Once a model was generated, it was used to produce a data set with $N=300$ members. The data set was then clustered using $k$-means. 


\section{B. Misassignment and overlap}

The centroids that resulted from clustering do not perfectly correspond to the probability vectors used to generate the data set. Each centroid was associated with the group in the probability model with the nearest probability vector. Since the data set was artificially generated, each member of the data set was unambiguously generated as part of some subgroup. The resulting clustered partition of the data set might not place members in the cluster associated with the group from which the member was generated. The fraction of students placed in the incorrect cluster will be called the misassignment rate $M$.

The closer two groups are together, the higher the probability a member generated from one group will be placed in a cluster associated with a different group.

The symmetric vectors explored in Sec. IV C and presented in Fig. 7 were used to investigate the relationship of overlap and misassignment. For each system in Fig. 7, 100 data sets were produced and clustered using the $k$-means algorithm. For each figure, the parameter $a$, the overlap $V$, and the average misassignment rate $M$ for the clustered models are reported. The misassignment rate increases with the overlap. An overlap of $V=0.25(a=0.7)$ shows little visual evidence of two distinct peaks.

The misassignment rate $M$ for models with different numbers of clusters is difficult to compare because the upper bound on $M$ changes with the number of clusters $N_{c}$. If data are randomly assigned to two clusters of equal size, half on average would be assigned to the wrong cluster, $M=\frac{1}{2}$. If data are randomly assigned to five clusters, the expected misassignment rate is $80 \%$. To compensate for this difference, consider a normalized misassignment rate $M_{N}$,

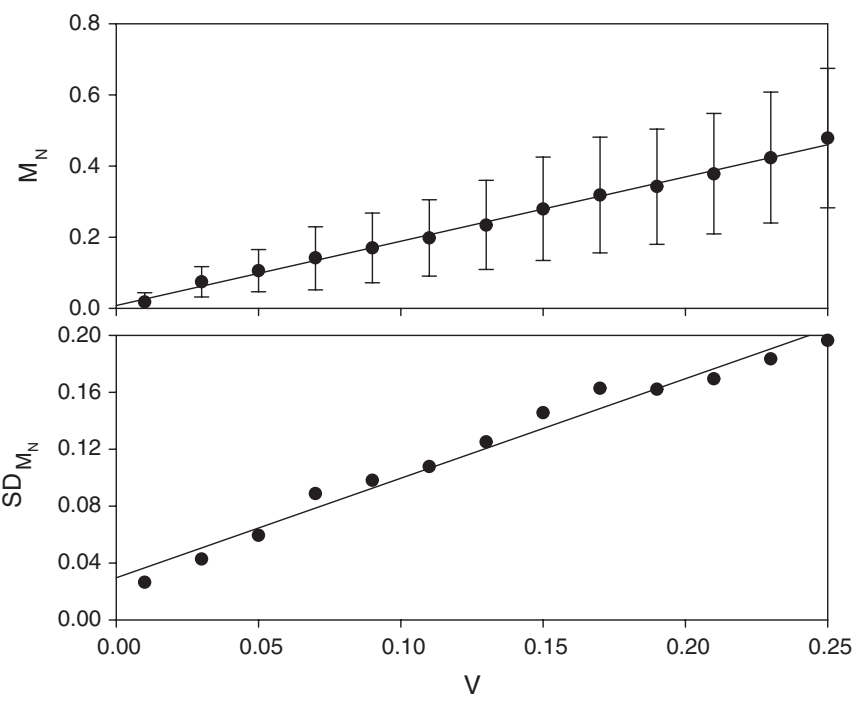

FIG. 10. Normalized misassignment rate $M_{N}$ versus overlap $V$, and the standard deviation in normalized misassignment rate $S D_{M_{N}}$ versus overlap.

$$
M_{N}=\frac{M}{1-1 / N_{c}} .
$$

The results of clustering the 400000 generated data sets are shown in Fig. 10. To generate this figure, the data were separated into segments of width $\Delta V=0.02$. The average normalized misassignment and the standard deviation of the normalized misassignment were calculated for each segment. The normalized misassignment grows fairly linearly with overlap for overlaps from $V=0.0$ to 0.25 . This linear trend does not continue beyond $V=0.25$ because the misassignment begins to saturate as the overlap grows. Examination of Fig. 7 shows that at $V=0.25$ the symmetric vector's probability distribution exhibits little visual evidence of the existence of two clusters. The data are well fitted by Eq. (21) relating the normalized misassignment to the overlap and by Eq. (22), which relates the standard deviation in the normalized misassignment rate $S D_{M_{N}}$ to the overlap:

$$
\begin{array}{cc}
M_{N}=1.91 V+0.0085 & \left(R^{2}=0.996\right), \\
S D_{M_{N}}=0.701 V+0.0293 & \left(R^{2}=0.977\right)
\end{array}
$$

\section{Effect of $N$}

All previous analysis was performed for data sets containing $N=300$ members. The 2006 course data from the University of Arkansas contains from 149 to 200 students; therefore, the effect of $N$ on the misassignment rate must be understood.

To allow prediction of the effect of $N$ on the misassignment rate, a variety of linear models were fit to randomly generated data sets that varied $N$ from 30 to 300.400000 random models were generated using the same set of strategies detailed in Sec. VIB. To extract the dependence of the normalized misassignment rate and its standard deviation on $N$ and $V$, the data were divided into regions of width $\Delta N=30$ in $N$ and $\Delta V=0.02$ in $V$. The average and standard deviation for $M_{N}$ was calculated for each region and a linear fit yielded

$$
M_{N}=1.90 V-0.103\left(\frac{N}{300}\right)+0.072 \quad\left(R^{2}=0.95\right)
$$

and

$S D_{M_{N}}=0.440 \mathrm{~V}-0.025\left(\frac{N}{300}\right)+0.083 \quad\left(R^{2}=0.76\right)$.

Since both $V$ and $N / 300$ have approximately the same range for the experiment, the size of their coefficients can be compared to evaluate the relative importance of overlap and $N$. The overlap has a much stronger influence on the normalized misassignment rate than the number of students in the sample. A nonlinear model adding an 
$N V$ term was also fit, but this addition had very little effect on $R^{2}$. For a $10 \%$ overlap, the normalized misassignment rate changes from $M_{N}=0.25$ at $N=30$ to $M_{N}=0.16$ at $N=300$.

\section{Centroid and vector error}

Cluster models do not perfectly locate the centroids or group population fractions of the underlying model. The average distance between the centroid found by $k$-means and the probability vector used to generate the random model can be calculated and will be called the "centroid error" $E_{c}$. A linear regression of centroid error against the overlap yielded

$$
E_{c}=0.495 V+0.036 \quad\left(R^{2}=0.38\right) .
$$

The centroid error also grows with overlap, as does its standard deviation.

The average error in the group population fractions $E_{g}$ can be calculated as the distance between the computed group population fractions and the model population fractions treated as vectors. This also increases with overlap and a linear regression of $E_{g}$ on $V$ yields Eq. (26). The standard deviation of $E_{g}$ also increases with overlap,

$$
E_{g}=0.295 \mathrm{~V}+0.020 \quad\left(R^{2}=0.21\right) .
$$

Note that the $R^{2}$ for the above equations is much smaller than other $R^{2}$ values reported. More investigation is required to understand why these quantities are so much harder to predict.

\section{CLUSTERING OF COURSE DATA}

The Spring and Fall 2006 data from the University of Arkansas were clustered using the $k$-means algorithm, and cluster models were extracted with $2-5$ clusters. The overlap $V$, predicted misassignment rate $M$, and $R^{2}$ are shown in Table II. The misassignment rate was calculated using Eqs. (20) and (23).

Examining the distributions in Fig. 7, one can see that the visual evidence for two clusters existing in the data set largely vanishes between $a=0.75$ and $a=0.7$, or in terms of misassignment rate between $M=0.07$ and $M=$ 0.13 . As such, we will use $M=0.10$ as a reasonable cutoff for a well-resolved cluster model. Only the twocluster models for the Spring 2006 posttest, Fall 2006 pretest, and the Fall 2006 posttest and the two-cluster and three-cluster cluster models for the Spring 2006 pretest meet the requirement that $M \leq 0.10$. The threecluster model for the Fall 2006 posttest with $M=0.117$ nearly meets the criterion. The group population fractions and probability vectors for these models are presented in Table III along with the group error $E_{g}$, centroid error $E_{c}$, and consistency $C$.

The two-cluster models shown in Table III are all of a similar character with probability vectors that are strongly
TABLE II. Clustering of pretest and posttest data. $N$ is the number of students, $N_{c}$ the number of clusters, $V$ the maximum pairwise overlap of the probability vectors, and $M$ the predicted

\begin{tabular}{|c|c|c|c|c|}
\hline \multicolumn{5}{|c|}{ Spring 2006 pretest } \\
\hline$N$ & $N_{c}$ & $V$ & $M$ & $R^{2}$ \\
\hline 149 & 2 & 0.010 & 0.020 & 0.69 \\
\hline 149 & 3 & 0.062 & 0.092 & 0.82 \\
\hline 149 & 4 & 0.160 & 0.244 & 0.87 \\
\hline 149 & 5 & 0.160 & 0.260 & 0.91 \\
\hline \multicolumn{5}{|c|}{ Spring 2006 posttest } \\
\hline$N$ & $N_{c}$ & $V$ & $M$ & $R^{2}$ \\
\hline 177 & 2 & 0.001 & 0.007 & 0.82 \\
\hline 177 & 3 & 0.128 & 0.170 & 0.89 \\
\hline 177 & 4 & 0.134 & 0.200 & 0.93 \\
\hline 177 & 5 & 0.281 & 0.436 & 0.95 \\
\hline \multicolumn{5}{|c|}{ Fall 2006 pretest } \\
\hline$N$ & $N_{c}$ & V & $M$ & $R^{2}$ \\
\hline 201 & 2 & 0.005 & 0.006 & 0.76 \\
\hline 201 & 3 & 0.211 & 0.269 & 0.86 \\
\hline 201 & 4 & 0.228 & 0.327 & 0.89 \\
\hline 201 & 5 & 0.321 & 0.491 & 0.91 \\
\hline \multicolumn{5}{|c|}{ Fall 2006 posttest } \\
\hline$N$ & $N_{c}$ & $V^{1}$ & $M$ & $R^{2}$ \\
\hline 195 & 2 & 0.001 & 0.004 & 0.87 \\
\hline 195 & 3 & 0.090 & 0.117 & 0.92 \\
\hline 195 & 4 & 0.154 & 0.224 & 0.94 \\
\hline 195 & 5 & 0.159 & 0.245 & 0.96 \\
\hline
\end{tabular}
misassignment rate.

weighted in a single component with one vector with a high probability of selecting the correct answer and the other vector with a strong probability of selecting a specific incorrect answer. This observation is in agreement with research showing that some students leave college science courses with strongly held misconceptions $[1-3,39]$.

In both cases, the three-cluster models introduce a third group with a relatively small group population fraction where the third group has a substantial probability of selecting either the correct answer or one of the distracters. This group selects either a Newtonian or a non-Newtonian answer based on context. This further supports research showing that some students can hold both a correct and an alternate model at the same time and apply them based on the contextual features of the situation [10-12,14]. Note that in the $\vec{p}_{3}$ vector for the Spring 2006 pretest threecluster model, the largest component is neither the correct answer nor the dominant misconception, but is a third component. This is the result of a differentiated response for a small subgroup of students to the problems using the soft contexts.

Table III also reports the centroid and group error along with misassignment rate; to put these errors in context, the 
TABLE III. Group population fractions and vectors. Correct answer shown in bold face. Note that some vectors do not add to 1 because of accumulated rounding error. $C$ is the course consistency as defined in Eq. (6), $E_{c}$ is the centroid error, and $E_{g}$ the group error.

\begin{tabular}{|c|c|}
\hline \multicolumn{2}{|c|}{$\begin{array}{l}\text { Spring } 2006 \text { pretest-two-cluster } \\
E_{c}=0.04, E_{g}=0.02, C=0.64\end{array}$} \\
\hline $\begin{array}{l}\gamma_{1}=0.49 \\
\gamma_{2}=0.51\end{array}$ & $\begin{array}{l}\vec{p}_{1}=(0.01, \mathbf{0 . 1 0}, 0.02,0.84,0.03) \\
\vec{p}_{2}=(0.13, \mathbf{0 . 7 3}, 0.02,0.08,0.04) \\
\text { retest-three-cluster } \\
=0.04, C=0.68\end{array}$ \\
\hline $\begin{array}{l}\gamma_{1}=0.39 \\
\gamma_{2}=0.48 \\
\gamma_{3}=0.13\end{array}$ & $\begin{array}{l}\vec{p}_{1}=(0.04, \mathbf{0 . 8 6}, 0.02,0.07,0.01) \\
\vec{p}_{2}=(0.01, \mathbf{0 . 1 0}, 0.02,0.85,0.01) \\
\vec{p}_{3}=(0.40, \mathbf{0 . 2 9}, 0.05,0.11,0.16) \\
2006 \text { posttest } \\
=0.02, C=0.87\end{array}$ \\
\hline $\begin{array}{l}\gamma_{1}=0.86 \\
\gamma_{2}=0.14\end{array}$ & $\begin{array}{l}\vec{p}_{1}=(0.02, \mathbf{0 . 9 4}, 0.01,0.03,0.01) \\
\vec{p}_{2}=(0.05, \mathbf{0 . 0 8}, 0.00,0.86,0.01) \\
006 \text { pretest } \\
=0.02, C=0.69\end{array}$ \\
\hline $\begin{array}{l}\gamma_{1}=0.25 \\
\gamma_{2}=0.75\end{array}$ & $\begin{array}{l}\vec{p}_{1}=(0.08,0.01,0.01,0.02, \mathbf{0 . 8 7}) \\
\vec{p}_{2}=(0.81,0.04,0.01,0.08, \mathbf{0 . 0 6}) \\
\text { sttest-two-cluster } \\
=0.02, C=0.83\end{array}$ \\
\hline $\begin{array}{l}\gamma_{1}=0.61 \\
\gamma_{2}=0.39\end{array}$ & $\begin{array}{l}\vec{p}_{1}=(0.04,0.01,0.00,0.00, \mathbf{0 . 9 5}) \\
\vec{p}_{2}=(0.84,0.03,0.02,0.04, \mathbf{0 . 0 7}) \\
\text { ttest-three-cluster } \\
=0.05, C=0.85\end{array}$ \\
\hline $\begin{array}{l}\gamma_{1}=0.30 \\
\gamma_{2}=0.11 \\
\gamma_{3}=0.59\end{array}$ & $\begin{array}{l}\vec{p}_{1}=(0.93,0.03,0.00,0.02, \mathbf{0 . 0 1}) \\
\vec{p}_{2}=(0.50,0.05,0.05,0.07, \mathbf{0 . 3 3}) \\
\vec{p}_{3}=(0.03,0.00,0.00,0.00, \mathbf{0 . 9 6})\end{array}$ \\
\hline
\end{tabular}

maximum distance between two $\vec{p}$ is $\sqrt{2}$. For three vectors, where one of the vectors is at the center of the other two as is approximately the case in the three-cluster models, the distance between the nearest clusters is $1 / \sqrt{2}$. The twocluster model with the maximum overlap was the Spring 2006 pretest model with $V=0.01$. At this overlap, Eq. (25) predicts a centroid error of $E_{c}=0.04$. The distance between the two centroids in this model is 0.99 , so the centroid error is $4 \%$ of the intercentroid distance. For the three-cluster models, the minimum intercentroid distance is 0.54 at an overlap of $V=0.09$ in Fall 2006, which yields a predicted error of $E_{c}=0.08,15 \%$ of the intercentroid distance. Therefore, the error in locating the centroid can become important as overlap increases or as the intercentroid distance decreases. Comparison of the group errors with the group population fractions shows that group error is also particularly important in the smaller groups in the three-cluster models.
Using the results of the previous section, the subgroup models extracted by $k$-means have predicted misassignment rates less the 10\% (excluding the three-cluster Fall 2006 posttest model) and acceptable centroid and group errors. The predicted overlap indicates that the extracted subgroups should be well separated from one another.

\section{DISCUSSION}

Four research questions were proposed in the introduction. Each will be discussed in turn.

Does the consistency of students' answers evolve over the course of an exam?

The pairwise consistency plotted in Fig. 2 shows very little change over the course of the exam. Since the consistency evolves little, the experiments presented in the Introduction that used paired problems should produce results that are not biased by changes in the student caused by the exam. This result also lends support for the validity of experiments using many contextually related questions $[11,13,19]$. The experiment presented in this paper spreads seven questions through a 36 question test while other work used a much higher density of contextually related questions, using exams with either nine consecutive contextually related questions [19] or eight questions in a tenquestion test $[11,13]$. More research is needed varying the length of the test before it could be concluded that multiple-question experiments with a much higher density of context-related questions also do not suffer from an evolution of consistency.

Table I presents the overall consistency $C$ for each data set. The students are quite consistent on both problems at the beginning of the course with $C=0.71$ and 0.72 . This consistency is commensurate with the consistency observed by Palmer [13] for college students postinstruction. The consistency on these problems for the course studied increases to $C=0.89$ and 0.86 postinstruction. The higher consistency produced by the course studied than that observed by Palmer could be a result of the problem selection (Palmer used Newton's first law), the population of students, the time between instruction and testing, or the inquiry-based nature of the course, among other factors. This observation of increased consistency postinstruction lends further support to studies finding that students' consistent application of both the correct and alternate models increases with instruction $[13,14]$.

While not directly related to the issue of the evolution of consistency, the experiment used a range of contexts from the traditional "hard" contexts used in most physics problems to "softer" contexts using balloons and pillows. As seen in Table I, these soft contexts did produce effects on consistency and problem average, but only at about the $10 \%$ level. This is a smaller effect than observed in previous studies [19], but the previous studies were done with high school students and this study is performed with college students. Students emerging from the 2006 courses 
were not substantially constrained to traditional physics contexts for their application of Newtonian principles. The effects of soft contexts showed most strongly in problem consistency; only the balloon context in the Spring 2006 posttest showed a substantially different score than that of the other problems. Three of the four softer contexts had noticeably lower consistency in the pretest. These differences mostly disappeared with instruction except once again for the balloon context. These observations support work showing that contextual sensitivity decreases with instruction $[6-8,13,14]$. The difference in the responses between the soft and hard physical contexts supports the observations of Liu and MacIsaac [15] that familiarity is an important variable in the consistent application of alternate models.

How can a data set containing the responses to multiple, context-related questions be used to subdivide a class into intellectually similar subgroups?

Model analysis and cluster analysis were compared using models with two subgroups. Model analysis produced good results in the limit when one subgroup contained the majority of students $\left(\gamma_{1} \approx 1\right)$ or when the probability vectors of the two subgroups were orthogonal. Away from these limits, the population fractions calculated by model analysis diverged from the fractions input into the models. The divergence was most severe when the two subgroups were similar in size, $\gamma_{1}=\frac{1}{2}$ and $\gamma_{2}=\frac{1}{2}$.

The cluster analysis algorithm investigated, $k$-means, correctly extracted the group population fractions over the range where visual inspection suggested subdivision into clusters was reasonable. Using the vectors extracted by $k$-means in Table III to estimate the relative error produced by model analysis generated small errors $(<5 \%)$ for only one of the four data sets. Since the errors predicted for model analysis were substantial for the studied course, cluster analysis was used to extract the subgroup structure of the course data. Methods were developed to characterize the quality of the models produced by cluster analysis using the overlap $V$. Application of these methods showed that the clustering algorithm produced subgroup population fractions and probability vectors with acceptable errors. As such, $k$-means clustering is an efficacious method of examining the subgroup structure of student understanding. Since model analysis overestimates the differences in $\gamma_{i}$ in many cases, it is possible that the large population fractions reported in previous research are artificially high.

Once a model subdividing a class into groups is constructed, how does one evaluate the quality of the model?

The identification of the centroids of the clusters as probability vectors allowed the introduction of a new method for characterizing the quality of a cluster model using the overlap $V$ between the probability distributions of pairs of probability vectors. The overlap $V$, which can be directly calculated from the probability vectors, allowed the prediction of the amount of misassignment, centroid, and group error in the cluster models. Balancing misassignment rate $M$ with $R^{2}$ allowed the selection of wellresolved cluster models.

It is possible that the correct underlying model does not allow clustering into well-resolved models. This would appear as a large $R^{2}$ for a set of centroids with high overlap. This situation undoubtedly occurs in some classrooms, but was not present in the course data clustered above.

What are the subgroup models of the Spring 2006 and Fall 2006 University Physics courses at the University of Arkansas?

Two semesters of pretest and posttest data were clustered using data taken at the University of Arkansas as shown in Table III. While the Spring 2006 pretest and the Fall 2006 posttest showed evidence for a three-cluster model where the third cluster contained students who had substantial probability of selecting one of two choices, the group population fractions for these clusters were small, 0.11 and 0.13 . Therefore, in the course studied there is little evidence for a substantial population of students applying both the correct model and an alternate model in different contextual situations. Though small, the existence of these three-cluster models shows that some students can be moved to a mixed state by a physics course (almost all students entering the course have had some physics instruction, so the pretest data can be viewed as the result of some previous physics course). This lends further support for the observations of Rosenblatt and Heckler showing that for some concepts students learn by passing through an intermediate, partially correct state [40]. Their work also strongly suggests that on some questions, but not all questions, three subgroups should be expected.

In all cases, most students' state of knowledge on the two questions was described by the two-cluster probability vectors. The two-cluster models featured one group that with high probability $(p>0.7)$ answered correctly, and one group that with high probability $(p>0.8)$ answered with the same incorrect answer. For the group that consistently selected the incorrect model, the consistency of selecting that model increased slightly with instruction for both semesters. This supports research showing that the consistency of application of both the correct model and incorrect models increases with instruction $[13,14]$.

The students entering the Spring 2006 course were approximately evenly divided between the groups on the static force question tested. These students left instruction predominately in the group that selected the correct answer $\gamma=0.86$ with a small number retaining persistent misconceptions. In Fall 2006, using a Newton's third law question involving systems in motion, $75 \%$ of the incoming students consistently selected an incorrect answer. This number dropped to $30 \%$ after instruction; even with inquiry-based instruction a substantial subpopulation was still consistently applying an incorrect model for this type of problem. 
The measurement of consistency $C$ presented in Table I substantially improves the characterization of the course over that provided by the average scores alone. The subgroup models presented in Table III further refine this characterization. Focusing on the Fall 2006 data, with no measure of consistency, the average score of $S=26 \%$ observed for the Fall 2006 pretest might be taken as a group of students that simply had no understanding of the problem. With a measured consistency of $C=0.72$, the course is shown to be formed of students who answer incorrectly because of strongly held alternate models. The subgroup model presented in Table III further refines this picture, showing a class divided into two groups, one forming one-quarter of the class that answers correctly $87 \%$ of the time and one forming three-quarters of the class that answers incorrectly-in the same way- $81 \%$ of the time. There is no substantial group of students who answer uncertainly entering the course in Fall 2006.

The effect of instruction is multifaceted. Using the three-cluster model for Fall 2006, the group answering consistently correctly expanded from $25 \%$ of the class to $59 \%$ of the class, and the consistency of answering correctly on each question also increased from $p_{5}=0.87$ to $p_{5}=0.96$ (the fifth response is correct). The group of students that answer consistently incorrectly shrank from $75 \%$ to $30 \%$ of the class. The consistency of these students in selecting one of the incorrect responses (the first response) also increased from $p_{1}=0.81$ to $p_{1}=0.93$. These students seem even more entrenched in the incorrect model postinstruction. The course also generated a small subpopulation (11\% of the students) who moved from consistently applying a misconception to applying that misconception and the correct model based on the context of the question. The subgroup model gives a much more detailed picture of the class over the score or the overall consistency alone in terms of the numbers of students left in each of these three states, correct, incorrect, and uncertain, and the details of the evolution of these states. This additional detail opens up the possibility of studies to determine how different modes of instruction affect the production of the third subgroup or can modify the rate of consistent application of the alternate models.

\section{IMPLICATIONS FOR INSTRUCTION}

The measurement above gives substantial additional information about a course, but the results were restricted to two problem sets for a single course. The specific implications for the instruction for the course studied will be discussed, as well as what different measurements would indicate for other courses.

The course studied produced significant progress in students' application of Newton's laws using a design that mixed traditional lecture with interactive laboratories. Most students leaving the courses could be divided into two groups: one group that consistently applied the Newtonian force concept correctly and one group that fairly consistently applied a single misconception. The size of the group of students with persistent misconceptions is $14 \%$ for the static force problem examined in the Spring 2006 semester and 30\% for the dynamic Newton's third law problem in Fall 2006 (again using the threecluster model). The students in these groups have not been moved significantly toward a Newtonian force concept on these concepts by the course; in fact, students in these groups answer incorrectly somewhat more consistently on the posttest than on the pretest. For these students, the instruction in the course, particularly the activities in the laboratory, have been completely ineffective in moving the students toward the correct model. Within the limits of instructional time, the concepts represented by these questions need to be revisited and new activities constructed to allow these students to confront their misconceptions. The laboratory already contains hands-on activities about the dynamical application of Newton's third law; these activities will have to be revisited and enhanced.

While most students in the courses studied were contained in two subgroups, different outcomes could have been observed for different courses and different problem choices. Two alternate outcomes are of particular interest: in some courses, it should be possible to observe a softening of the consistency of the group holding alternate conceptions or the production of a substantial third subgroup that holds both the correct model and the alternate model. Both of these outcomes would produce subpopulations of students who apply both the correct model and an incorrect model depending on the situation. Work on characterizing the subgroup structure of classes [34] and understanding the features of the misconceptions that influence that structure [35] are evolving, so specific implications for instruction for the above cases do not yet exist. It would be very interesting to determine the features of a course that produces this shift in consistency. Furthermore, while it seems likely that intensive, laboratory-based interventions will be required to move students in the course studied away from the Newton's third law misconception, perhaps alternate methods would be useful in a course that produces students holding both models simultaneously. It would also be very interesting to study how the knowledge states of these students evolve over time. Would the students fall back to applying the misconception consistently or move toward correctly applying Newton's third law? It is only through the production of reliable methods to extract the subgroup structure of a class that these important instructional issues can be investigated.

Consistency $C$ provides an important additional statistic beyond the average score on the problem $S$ to aid in understanding an incoming student population and the results of instruction. Since $C$ evolves little over a 
multiple-question exam, a set of paired questions can be used to estimate $C$ on a given concept. These questions may be drawn from standard instruments such as the FCI or Force and Motion Conceptual Evaluation [41] in many cases [34] and $C$ should be easily calculable by working instructors. The additional information provided by the cluster models requires substantially more effort and probably requires more time than is available to a working instructor, and therefore will remain primarily a research tool. It is our hope that versions of standard conceptual evaluations containing multiple contextually related problems can be created and made available along with resources to do the clustering at Web sites maintained by the physics community.

\section{FUTURE}

The $k$-means algorithm is only one of many clustering algorithms. The modeling of a cluster by a single parameter, its center, contains the assumption of spherical clusters. The probability vectors found when clustering the course data do not produce spherical clusters; the clusters are heavily weighted in two components and produce clusters that tend to spread along a line. In the future, clustering algorithms that can account for nonspherical cluster shapes will be investigated.

The experiment performed could be repeated with any FCI question to determine if the observation that the students were either left in a state where they consistently selected the correct answer most of the time or consistently selected the wrong answer was a general feature of the course or only a feature of the two questions studied. It would also be interesting to extend this research to other institutions to see if probability vectors with the same general qualitative features were produced.

It would be very interesting to repeat this analysis for different forms of instruction and determine how the class subgroup structure is impacted by instructional methods.

\section{CONCLUSION}

The consistency in answering conceptual Newtonian mechanics questions evolved very little over seven matched questions; therefore, the consistency measured by a set of paired questions should be a good estimate of the overall consistency. The $k$-means algorithm is an effective mechanism for extracting the underlying subgroups in student data. The statistical overlap of the probability distributions of the student responses in the class subgroups is a highly useful statistic for characterizing these models. The cluster models extracted from data taken at the University of Arkansas suggested that consistency increased during instruction in an inquiry-based course, but a subset of students were left consistently applying a nonNewtonian model.

\section{ACKNOWLEDGMENTS}

This work is supported in part by the National Science Foundation as part of the evaluation of improved learning for the Physics Teacher Education Coalition, PHY0108787.

\section{APPENDIX}

Original FCI problem 29-Spring 2006 An empty office chair is at rest on a floor. Consider the following forces:

A. a downward force of gravity.

B. an upward force exerted by the floor.

C. a net downward force exerted by the air.

Which of the forces is (are) acting on the office chair?

(a) A only

(b) A and B

(c) $\mathrm{B}$ and $\mathrm{C}$

(d) A, B, and C

(e) None of the forces. (Since the chair is at rest, there are no forces acting upon it.)

Example of transformed FCI problem 29-Spring $2006 \mathrm{~A}$ car is at rest in your driveway. Consider the following forces:

A. a downward force of gravity.

B. an upward force exerted by the driveway.

C. a net downward force exerted by the air.

Which of the forces is (are) acting on the car?

(1) A only

(2) A and B

(3) B and $C$

(4) A, B, and C

(5) None of the forces. (Since the car is at rest, there are no forces acting upon it.)

Example of original FCI Problem 4-Fall 2006 A large truck collides head-on with a small compact car. During the collision

(1) the truck exerts a greater amount of force on the car than the car exerts on the truck.

(2) the car exerts a greater amount of force on the truck than the truck exerts on the car.

(3) neither exerts a force on the other, the car gets smashed simply because it gets in the way of the truck.

(4) the truck exerts a force on the car but the car does not exert a force on the truck.

(5) the truck exerts the same amount of force on the car as the car exerts on the truck.

Example of transformed FCI problem 4-Fall 2006 A bulldozer collides head-on with a bicycle. During the collision 
(1) the bulldozer exerts a greater amount of force on the bicycle than the bicycle exerts on the bulldozer.

(2) the bicycle exerts a greater amount of force on the bulldozer than the bulldozer exerts on the bicycle.

(3) neither exerts a force on the other, the bicycle gets smashed simply because it gets in the way of the bulldozer.
(4) the bulldozer exerts a force on the bicycle but the bicycle does not exert a force on the bulldozer.

(5) the bulldozer exerts the same amount of force on the bicycle as the bicycle exerts on the bulldozer.
[1] Richard R. Hake, Interactive-engagement versus traditional methods: A six-thousand-student survey of mechanics test data for introductory physics courses, Am. J. Phys. 66, 64 (1998).

[2] David Hestenes, Malcolm Wells, and Gregg Swackhamer, Force concept inventory, Phys. Teach. 30, 141 (1992).

[3] Ibrahim Abou Halloun and David Hestenes, The initial knowledge state of college physics students, Am. J. Phys. 53, 1043 (1985).

[4] Andrea A. diSessa, Knowledge in pieces, in Constructivism in the Computer Age, edited by George Forman and Peter B. Pufall (Erlbaum, Hillsdale, NJ, 1988).

[5] Michelene T. H. Chi and James D. Slotta, The ontological coherence of intuitive physics, Cogn. Instr. 10, 249 (1993).

[6] Gail S. Gliner, College students' organization of mathematics word problems in relation to success in problem solving, School Sci. Math. 89, 392 (1989).

[7] Gail S. Gliner, College students' organization of mathematics word problems in terms of mathematical structure vs. surface structure. School Sci. Math. 91, 105 (1991).

[8] Michelene T. H. Chi, Paul J. Feltovich, and Robert Glaser, Categorization and representation of physics problems by experts and novices, Cogn. Sci. 5, 121 (1981).

[9] Patrick B. Kohl and Noah D. Finkelstein, Patterns of multiple representation use by experts and novices during physics problem solving, Phys. Rev. ST Phys. Educ. Res. 4, 010111 (2008).

[10] Elizabeth Engel Clough and Rosalind Driver, A study of consistency in the use of students' conceptual frameworks across different task contexts, Sci. Educ. 70, 473 (1986).

[11] David H. Palmer, Measuring contextual error in the diagnosis of alternative conceptions in science, Issues Educ. Res. 8, 65 (1998) [http://www.iier.org.au/iier8/ palmer.html].

[12] Salomon F. Itza-Ortiz, Sanjay Rebello, and Dean Zollman, Students' models of Newton's second law in mechanics and electromagnetism, Eur. J. Phys. 25, 81 (2004).

[13] David Palmer, The effect of context on students' reasoning about forces, Int. J. Sci. Educ. 19, 681 (1997).

[14] M. Finegold and P. Gorsky, Students' concepts of force as applied to related physical systems: A search for consistency, Int. J. Sci. Educ. 13, 97 (1991).

[15] Xiufeng Liu and Dan MacIsaac, An investigation of factors affecting the degree of naive impetus theory application, J. Sci. Educ. Technol. 14, 101 (2005).
[16] Nanjundiah Sadanand and Joseph Kess, Concepts in force and motion, Phys. Teach. 28, 530 (1990).

[17] John Stewart, Heather Griffin, and Gay Stewart, Context sensitivity in the Force Concept Inventory, Phys. Rev. ST Phys. Educ. Res. 3, 010102 (2007).

[18] Lonnie J. Rennie and Lesley H. Parker, Placing physics problems in real-life context: Students' reactions and performance, Aust. Sci. Teachers J. 42, 55 (1996) [http://www.eric.ed.gov/ERICWebPortal/detail?accno= EJ530089].

[19] David H. Palmer, Investigating the relationship between students' multiple conceptions of action and reaction in cases of static equilibrium, Res. Sci. Technol. Educ. 19, 193 (2001).

[20] Lillian C. McDermott, Mark L. Rosenquist, and Emily H. van Zee, Student difficulties in connecting graphs and physics: Examples from kinematics, Am. J. Phys. 55, 503 (1987).

[21] David E. Meltzer, Relation between students' problemsolving performance and representational format, Am. J. Phys. 73, 463 (2005).

[22] Patrick B. Kohl and Noah D. Finkelstein, Student representational competence and self-assessment when solving physics problems, Phys. Rev. ST Phys. Educ. Res. 1, 010104 (2005).

[23] Patrick B. Kohl and Noah D. Finkelstein, Effects of representation on students solving physics problems: A fine-grained characterization, Phys. Rev. ST Phys. Educ. Res. 2, 010106 (2006).

[24] Antti Savinainen, Pasi Nieminen, Jouni Viiri, Jukka Korkea-aho, and Aku Talikka, FCI-based multiple choice test for investigating students' representational coherence, AIP Conf. Proc. 951, 176 (2007).

[25] Pasi Nieminen, Antti Savinainen, and Jouni Viiri, Force Concept Inventory-based multiple-choice test for investigating students representational consistency. Phys. Rev. ST Phys. Educ. Res. 6, 020109 (2010).

[26] Kenneth R. Koedinger and Mitchell J. Nathan, The real story behind story problems: Effects of representations on quantitative reasoning, J. Learn. Sci. 13, 129 (2004).

[27] Robert J. Dufresne, William J. Gerace, and William J. Leonard, Solving physics problems with multiple representations, Phys. Teach. 35, 270 (1997).

[28] Patrick B. Kohl and Noah D. Finkelstein, Effect of instructional environment on physics students representational skills, Phys. Rev. ST Phys. Educ. Res. 2, 010102 (2006). 
[29] Gita Taasoobshirazi and Martha Carr, A review and critique of context-based physics instruction and assessment, Educ. Res. Rev. 3, 155 (2008).

[30] Patricia Heller and Mark Hollabaugh, Teaching problem solving through cooperative grouping. Part 2: Designing problems and structuring groups, Am. J. Phys. 60, 637 (1992).

[31] Shih-Yin Lin and Chandralekha Singh, Using isomorphic problems to learn introductory physics, Phys. Rev. ST Phys. Educ. Res. 7, 020104 (2011).

[32] Chandralekha Singh, Assessing student expertise in introductory physics with isomorphic problems. I. Performance on nonintuitive problem pair from introductory physics, Phys. Rev. ST Phys. Educ. Res. 4, 010104 (2008).

[33] Chandralekha Singh, Assessing student expertise in introductory physics with isomorphic problems. II. Effect of some potential factors on problem solving and transfer, Phys. Rev. ST Phys. Educ. Res. 4, 010105 (2008).

[34] Lei Bao and Edward F. Redish, Model analysis: Representing and assessing the dynamics of student learning, Phys. Rev. ST Phys. Educ. Res. 2, 010103 (2006).

[35] Lei Bao, Kirsten Hogg, and Dean Zollman, Model analysis of fine structures of student models: An example with Newton's third law, Am. J. Phys. 70, 766 (2002).
[36] E. Mazur, Peer Instruction (Prentice-Hall, Englewood Cliffs, NJ, 1997).

[37] Lei Bao, Dynamics of Student Modeling: A Theory, Algorithms, and Applications to Quantum Mechanics, Ph.D. thesis, University of Maryland, 1999.

[38] J.B. MacQueen, Some methods for classification and analysis of multivariate observations, in Proceedings of the Fifth Berkeley Symposium on Mathematical Statistics and Probability, edited by L. M. Le Cam and J. Neyman (University of California Press, Berkeley, CA, 1967), pp. 281-297.

[39] Ibrahim Abou Halloun and David Hestenes, Common sense concepts about motion, Am. J. Phys. 53, 1056 (1985).

[40] Rebecca Rosenblatt and Andrew F. Heckler, Systematic study of student understanding of the relationships between the directions of force, velocity, and acceleration in one dimension, Phys. Rev. ST Phys. Educ. Res. 7, 020112 (2011).

[41] Ronald K. Thornton and David R. Sokoloff, Accessing student learning of Newton's laws: The Force and Motion Conceptual Evaluation and the evaluation of active learning laboratory and curricula, Am. J. Phys. 66, 338 (1998). 